\title{
Redox-Triggered Interconversion between Piperidine Chair Conformations in a $\mathrm{Cu}(\mathrm{I} / \mathrm{II})$ Complex
}

\author{
Jing Zhang and James W. Canary* \\ Department of Chemistry, New York University, New York, NY 10003 \\ Canary@nyu.edu
}

\begin{abstract}
All solvents and reagents were purchased from commercial sources and used as received. UV-visible spectra were acquired on an Agilent 8453 UV-vis spectrometer. Circular dichroism spectra were recorded on an AVIV model 202SF spectropolarimeter. NMR spectra were obtained on a Bruker AVANCE $400 \mathrm{MHz}$ spectrometer. Mass spectra were recorded on an Agilent 1100 LC-MS spectrometer. Melting points were taken in open tubes in a MelTemp II capillary melting point apparatus. Elemental analysis was performed by Quantitative Technologies, Inc. (Whitehouse, NJ). The calculation was performed on Spartan using the semi-empirical method PM3(tm) for copper complexes and the molecular mechanics method MMFF94 for free ligand.
\end{abstract}

2-Bromo-4-methyl-quinoline (2):

The compounds 2-hydroxy-4-methyl-quinoline $(8.4 \mathrm{~g}, 53 \mathrm{mmol})$ and phosphorous oxybromide (13.6 g, $47 \mathrm{mmol})$ were mixed under argon and preheated at $60^{\circ} \mathrm{C}$. The phosphorous oxybromide liquidified after $10 \mathrm{~min}$. The temperature was increased to $120^{\circ} \mathrm{C}$ and maintained for $1.5 \mathrm{~h}$. The mixture was cooled down and ice was added carefully. The suspension was poured into $300 \mathrm{~g}$ ice and basified with sodium 
hydroxide. The solid was collected by filtration and dried in the oven at $40^{\circ} \mathrm{C}$. The crude product was purified by silica chromatography using methylene chloride as eluent to give $9.2 \mathrm{~g}$ of white solid product. The yield was $88 \%$. Melting point: $78-79^{\circ} \mathrm{C} .{ }^{1} \mathrm{H}-\mathrm{NMR}\left(\mathrm{CDCl}_{3}\right.$, $400 \mathrm{MHz}): \delta=8.05(\mathrm{dd}, \mathrm{J}=8.3,1.3 \mathrm{~Hz}, 1 \mathrm{H}, \mathrm{Ar}-\mathrm{H}), 7.97(\mathrm{dd}, \mathrm{J}=8.4,1.3 \mathrm{~Hz}, 1 \mathrm{H}, \mathrm{Ar}-\mathrm{H})$, 7.74 (ddd, J = 8.4, 7.8, 1.4 Hz, 1H, Ar-H), 7.60 (ddd, J = 8.4, 7.8, $1.4 \mathrm{~Hz}, 1 \mathrm{H}$, Ar-H), 7.40 (s, $1 \mathrm{H}, \mathrm{Ar}-\mathrm{H}), 2.69\left(\mathrm{~s}, 3 \mathrm{H}, \mathrm{CH}_{3}\right) \cdot{ }^{13} \mathrm{C}-\mathrm{NMR}\left(\mathrm{CDCl}_{3}, 400 \mathrm{MHz}\right): \delta=148.34,147.18$, 141.94, 130.21, 129.26, 127.14, 126.80, 125.93, 123.95, 18.42. ESI-MS: m/z 222.2 (M $+\mathrm{H}^{+}$); calculated: 222.0. Anal. Calcd (mass\%) for $\mathrm{C}_{10} \mathrm{H}_{8} \mathrm{BrN}: \mathrm{C}, 54.08 ; \mathrm{H}, 3.63 ; \mathrm{N}, 6.31$. Found: C, 53.94; H, 3.64; N, 6.19.

(4-Methyl-quinolin-2-yl)-methanol (3):

To a solution of $\mathrm{n}$-BuLi (1.6 M in hexane, $17.2 \mathrm{~mL}, 27.5 \mathrm{mmol}$ ) in THF (15mL) was added dropwise a solution of bromo compound $2(5.5 \mathrm{~g}, 25 \mathrm{mmol})$ in $35 \mathrm{~mL}$ of THF at $-78^{\circ} \mathrm{C}$ under argon gas. After $30 \mathrm{~min}$, dry DMF $(2.7 \mathrm{~mL}, 35 \mathrm{mmol})$ was added and reacted for another $30 \mathrm{~min}$. Methanol $(25 \mathrm{~mL})$ and acetic acid $(2 \mathrm{~mL})$ were added until the solution turned orange in color. After sodium borohydride $(1.32 \mathrm{~g}, 35 \mathrm{mmol})$ was added, the solution was allowed to come to room temperature. The reaction was quenched carefully with saturated ammonium chloride. The residue left from removal of the solvent was partitioned between methylene chloride and water. The combined organic layer was dried by sodium sulfate and the product was further purified by silica chromatography (methylene chloride / ethyl acetate 10:1) affording a light yellow solid (3.67 g, 85\%). Melting point: $80-84^{\circ} \mathrm{C} .{ }^{1} \mathrm{H}-\mathrm{NMR}\left(\mathrm{CDCl}_{3}, 400 \mathrm{MHz}\right): \delta=8.08(\mathrm{~d}, \mathrm{~J}=8.2$ $\mathrm{Hz}, 1 \mathrm{H}, \mathrm{Ar}-\mathrm{H}), 7.98(\mathrm{~d}, \mathrm{~J}=8.3 \mathrm{~Hz}, 1 \mathrm{H}, \mathrm{Ar}-\mathrm{H}), 7.71(\mathrm{t}, \mathrm{J}=8.6 \mathrm{~Hz}, 1 \mathrm{H}, \operatorname{Ar}-\mathrm{H}), 7.55(\mathrm{t}, \mathrm{J}=$ $8.6 \mathrm{~Hz}, 1 \mathrm{H}, \mathrm{Ar}-\mathrm{H}), 7.12(\mathrm{~s}, 1 \mathrm{H}, \mathrm{Ar}-\mathrm{H}), 4.87\left(\mathrm{~s}, 2 \mathrm{H}, \mathrm{CH}_{2}\right), 4.55(\mathrm{~s}, \mathrm{~b}, 1 \mathrm{H}, \mathrm{OH}), 2.70(\mathrm{~s}, 3 \mathrm{H}$, $\left.\mathrm{CH}_{3}\right) \cdot{ }^{13} \mathrm{C}-\mathrm{NMR}\left(\mathrm{CDCl}_{3}, 400 \mathrm{MHz}\right): \delta=158.60,146.56,145.05,129.43,129.20,127.65$, 
126.09, 123.82, 118.92, 64.00, 18.80. ESI-MS: $\mathrm{m} / \mathrm{z} 174.3\left(\mathrm{M}+\mathrm{H}^{+}\right)$; calculated: 174.1 . Anal. Calcd (mass\%) for $\mathrm{C}_{11} \mathrm{H}_{11} \mathrm{NO}$ : C, 76.28; H, 6.40; N, 8.09. Found: C, 76.30; H, 6.26; N, 7.95 .

Methanesulfonic acid 4-methyl-quinolin-2-ylmethyl ester (4):

A solution of alcohol $3(3.51 \mathrm{~g}, 20.3 \mathrm{mmol})$ and triethylamine $(4.9 \mathrm{~mL}, 48 \mathrm{mmol})$ in $40 \mathrm{~mL}$ of methylene chloride was added slowly to $30 \mathrm{~mL}$ of a methylene chloride solution of methanesulfonyl chloride $(4.70 \mathrm{~g}, 41.0 \mathrm{mmol})$ at $-30^{\circ} \mathrm{C}$. The solution was stirred at this temperature for $3 \mathrm{~h}$ and then water was added for extraction. The organic layer was dried with sodium sulfate and the solvent was evaporated to give a brown solid product $(5.06 \mathrm{~g}, 99 \%)$. Melting point: $92-94^{\circ} \mathrm{C} .{ }^{1} \mathrm{H}-\mathrm{NMR}\left(\mathrm{CDCl}_{3}, 400 \mathrm{MHz}\right): \delta=$ $8.06(\mathrm{~d}, \mathrm{~J}=8.5 \mathrm{~Hz}, 1 \mathrm{H}, \mathrm{Ar}-\mathrm{H}), 8.01(\mathrm{~d}, \mathrm{~J}=8.4 \mathrm{~Hz}, 1 \mathrm{H}, \mathrm{Ar}-\mathrm{H}), 7.73(\mathrm{t}, \mathrm{J}=8.3 \mathrm{~Hz}, 1 \mathrm{H}, \mathrm{Ar}-$ H), $7.60(\mathrm{t}, \mathrm{J}=8.2 \mathrm{~Hz}, 1 \mathrm{H}, \mathrm{Ar}-\mathrm{H}), 7.42(\mathrm{~s}, 1 \mathrm{H}, \mathrm{Ar}-\mathrm{H}), 5.46\left(\mathrm{~s}, 2 \mathrm{H}, \mathrm{CH}_{2}\right), 3.12\left(\mathrm{~s}, 3 \mathrm{H}, \mathrm{CH}_{3}\right)$, $2.74\left(\mathrm{~s}, 3 \mathrm{H}, \mathrm{CH}_{3}\right) .{ }^{13} \mathrm{C}-\mathrm{NMR}\left(\mathrm{CDCl}_{3}, 400 \mathrm{~Hz}\right): \delta=153.47,147.38,146.00,129.80$, 129.78, 127.80, 126.99, 123.83, 120.29, 72.28, 38.09, 18.84. ESI-MS: m/z $252.3(M+$ $\mathrm{H}^{+}$); calculated: 252.1 .

\section{2-Azidomethyl-4-methyl-quinoline (5):}

Sodium azide $(2.60 \mathrm{~g}, 40 \mathrm{mmol})$ and 18-crown-6 $(0.21 \mathrm{~g}, 0.8 \mathrm{mmol})$ were mixed in $8 \mathrm{~mL}$ of dry acetonitrile and stirred for $30 \mathrm{~min}$. Then mesylate $4(5.03 \mathrm{~g}, 20 \mathrm{mmol})$ in $14 \mathrm{~mL}$ of $\mathrm{CH}_{3} \mathrm{CN}$ was added and stirred overnight. An insoluble solid was removed by filtration. The filtrate was condensed to give a brown oil residue which was subjected to silica gel chromatography (methylene chloride / ethyl acetate from 10:0 to 10:1). The reddish oily solid product $(3.37 \mathrm{~g})$ was collected with a yield of $85 \%$. ${ }^{1} \mathrm{H}-\mathrm{NMR}\left(\mathrm{CDCl}_{3}\right.$, $400 \mathrm{MHz}): \delta=8.07(\mathrm{~d}, \mathrm{~J}=8.5 \mathrm{~Hz}, 1 \mathrm{H}, \mathrm{Ar}-\mathrm{H}), 7.98(\mathrm{~d}, \mathrm{~J}=8.5 \mathrm{~Hz}, 1 \mathrm{H}, \mathrm{Ar}-\mathrm{H}), 7.71(\mathrm{t}, \mathrm{J}=$ 
8.3 Hz, $1 \mathrm{H}, \operatorname{Ar}-\mathrm{H}), 7.56(\mathrm{t}, \mathrm{J}=8.2 \mathrm{~Hz}, 1 \mathrm{H}, \mathrm{Ar}-\mathrm{H}), 7.30(\mathrm{~s}, 1 \mathrm{H}, \mathrm{Ar}-\mathrm{H}), 4.61\left(\mathrm{~s}, 2 \mathrm{H}, \mathrm{CH}_{2}\right)$, $2.71\left(\mathrm{~s}, 3 \mathrm{H}, \mathrm{CH}_{3}\right) .{ }^{13} \mathrm{C}-\mathrm{NMR}\left(\mathrm{CDCl}_{3}, 400 \mathrm{~Hz}\right): \delta=154.70,146.62,144.78,128.88$, 128.70, 126.61, 125.61, 122.84, 119.46, 55.36, 17.93. ESI-MS: $\mathrm{m} / \mathrm{z} 199.3\left(\mathrm{M}+\mathrm{H}^{+}\right)$; calculated: 199.1 .

(4-Methyl-quinolin-2-yl)-methylamine (6):

Azide 5 (2.63 g, $13.3 \mathrm{mmol}$ ) was dissolved in $30 \mathrm{~mL}$ of dry THF, and then triphenylphosphine $(3.83 \mathrm{~g}, 14.6 \mathrm{mmol})$ was added slowly. After the mixture was stirred for $2 \mathrm{~h}, 0.3 \mathrm{~mL}$ of water was added and the mixture was stirred overnight. The solvent was removed and the residue was partitioned between ether and $5 \% \mathrm{HCl}$. After removing the organic layer, the acidic aqueous layer was basified carefully with $\mathrm{NaOH}$ carefully and extracted with methylene chloride. The solvent was removed, dried over sodium sulfate, and concentrated to produce $2.17 \mathrm{~g}(95 \%)$ of reddish liquid product. ${ }^{1} \mathrm{H}$ NMR $\left(\mathrm{CDCl}_{3}, 400 \mathrm{MHz}\right): \delta=8.05(\mathrm{~d}, \mathrm{~J}=8.5 \mathrm{~Hz}, 1 \mathrm{H}, \mathrm{Ar}-\mathrm{H}), 7.97(\mathrm{~d}, \mathrm{~J}=8.3 \mathrm{~Hz}, 1 \mathrm{H}, \mathrm{Ar}-$ H), $7.69(\mathrm{t}, \mathrm{J}=8.3 \mathrm{~Hz}, 1 \mathrm{H}, \mathrm{Ar}-\mathrm{H}), 7.53(\mathrm{t}, \mathrm{J}=8.2 \mathrm{~Hz}, 1 \mathrm{H}, \mathrm{Ar}-\mathrm{H}), 7.25(\mathrm{~s}, 1 \mathrm{H}, \mathrm{Ar}-\mathrm{H}), 4.12$ (s, 2H, CH 2$), 2.70\left(\mathrm{~s}, 3 \mathrm{H}, \mathrm{CH}_{3}\right), 1.80\left(\mathrm{~s}, \mathrm{~b}, 2 \mathrm{H}, \mathrm{NH}_{2}\right) \cdot{ }^{13} \mathrm{C}-\mathrm{NMR}\left(\mathrm{CDCl}_{3}, 400 \mathrm{~Hz}\right): \delta=$ 161.79, 147.60, 144.63, 129.47, 129.17, 127.30, 125.77, 123.69, 120.51, 48.34, 18.72. ESI-MS: $\mathrm{m} / \mathrm{z} 173.3\left(\mathrm{M}+\mathrm{H}^{+}\right)$; calculated: 173.1 .

$N^{1}, N^{5}$-Dimethoxy- $N^{1}, N^{5}$-dimethylglutaramide (7): ${ }^{a}$

Glutaryl dichloride $(5.07 \mathrm{~g}, 30 \mathrm{mmol})$ and $\mathrm{N}$-O-dimethylhydroxylamine hydrochloride $(6.45 \mathrm{~g}, 66 \mathrm{mmol})$ were mixed in $100 \mathrm{~mL}$ of dry methylene chloride at $0^{\circ} \mathrm{C}$. After $14.6 \mathrm{~mL}$ of dry pyridine $(14.2 \mathrm{~g}, 180 \mathrm{mmol})$ was added dropwise, the mixture was warmed to room temperature and stirred for $3 \mathrm{~h}$. The insoluble salt was filtered and the filtrate was condensed. The residue was partitioned between brine and 1:1 ether / 
methylene chloride. The organic layer was dried over magnesium sulfate, and removal of solvent gave $6.41 \mathrm{~g}$ of brown oil in $98 \%$ yield. ${ }^{1} \mathrm{H}-\mathrm{NMR}\left(\mathrm{CDCl}_{3}, 400 \mathrm{MHz}\right): \delta=3.62(\mathrm{~s}$, $\left.3 \mathrm{H}, \mathrm{CH}_{3}\right), 3.10\left(\mathrm{~s}, 3 \mathrm{H}, \mathrm{CH}_{3}\right), 2.44\left(\mathrm{t}, \mathrm{J}=6.7 \mathrm{~Hz}, 4 \mathrm{H}, \mathrm{CH}_{2}\right), 1.88\left(\mathrm{p}, \mathrm{J}=7.2 \mathrm{~Hz}, 2 \mathrm{H}, \mathrm{CH}_{2}\right)$

1,5-Bis-(4-methyl-quinolin-2-yl)-pentane-1,5-dione (8):

To $18 \mathrm{~mL}$ of THF was added $\mathrm{n}$-BuLi (1.6M in hexane, $30 \mathrm{~mL}, 48 \mathrm{mmol})$, followed by the slow addition of $120 \mathrm{~mL}$ of a THF solution of $2(8.82 \mathrm{~g}, 40 \mathrm{mmol})$ at $-78^{\circ} \mathrm{C}$ under argon. The resulting dark solution was stirred for an additional $30 \mathrm{~min}$. Diamine 7 (4.14 $\mathrm{g}, 19 \mathrm{mmol}$ ) in $30 \mathrm{~mL}$ of THF was added and the mixture was warmed to room temperature and stirred overnight. The residue resulting from solvent removal was subjected to silica gel chromatography (methylene chloride / pet ether 1:3) to give a white solid product $(4.43 \mathrm{~g}, 61 \%)$. Melting point: $132-134^{\circ} \mathrm{C} .{ }^{1} \mathrm{H}-\mathrm{NMR}\left(\mathrm{CDCl}_{3}, 400 \mathrm{MHz}\right)$ : $\delta=8.13(\mathrm{~d}, \mathrm{~J}=8.5 \mathrm{~Hz}, 2 \mathrm{H}, \mathrm{Ar}-\mathrm{H}), 8.00(\mathrm{~d}, \mathrm{~J}=8.3 \mathrm{~Hz}, 2 \mathrm{H}, \mathrm{Ar}-\mathrm{H}), 7.96(\mathrm{~s}, 2 \mathrm{H}, \mathrm{Ar}-\mathrm{H}), 7.72$ (t, J = 8.3 Hz, 2H, Ar-H), $7.63(\mathrm{t}, \mathrm{J}=8.3 \mathrm{~Hz}, 2 \mathrm{H}, \mathrm{Ar}-\mathrm{H}), 3.56\left(\mathrm{t}, \mathrm{J}=7.3,4 \mathrm{H}, \mathrm{CH}_{2}\right.$ ), 2.74 (s, $\left.6 \mathrm{H}, \mathrm{CH}_{3}\right), 2.31\left(\mathrm{t}, \mathrm{J}=7.3 \mathrm{~Hz}, 2 \mathrm{H}, \mathrm{CH}_{2}\right) \cdot{ }^{13} \mathrm{C}-\mathrm{NMR}\left(\mathrm{CDCl}_{3}, 400 \mathrm{~Hz}\right): \delta=202.53,152.71$, $147.05,145.20,131.17,129.58,129.46,128.13,123.73,118.66,36.86,18.87,18.79$. ESI-MS: $\mathrm{m} / \mathrm{z} 383.4\left(\mathrm{M}+\mathrm{H}^{+}\right.$); calculated: 383.2. Anal. Calcd (mass\%) for $\mathrm{C}_{25} \mathrm{H}_{22} \mathrm{~N}_{2} \mathrm{O}_{2}$ : $\mathrm{C}$, $78.51 ; \mathrm{H}, 5.80 ; \mathrm{N}, 7.32$. Found: C, 78.51; H, 5.50; N, 7.25.

(1S,5S)-1,5-Bis-(4-methyl-quinolin-2-yl)-pentane-1,5-diol (9):

To $40 \mathrm{~mL}$ of a THF solution of diketone $8(3.50 \mathrm{~g}, 9.2 \mathrm{mmol})$ was added $60 \mathrm{~mL}$ of a THF solution of (-)-DIP-Cl $(6.47 \mathrm{~g}, 20.2 \mathrm{mmol})$ at $-78^{\circ} \mathrm{C}$ under argon. After stirring for 2 $\mathrm{h}$, the solution was warmed to room temperature and stirred overnight. A solution of $10 \%$ $\mathrm{HCl}$ was added to quench the reaction, followed by stirring for another hour. After THF was removed, the aqueous portion was washed twice with ether and once with 
methylene chloride. The aqueous layer was basified with saturated $\mathrm{Na}_{2} \mathrm{CO}_{3}$ and extracted with methylene chloride. Removal of the solvent after drying over sodium sulfate gave $3.36 \mathrm{~g}(95 \%)$ of product as a white solid. Melting point: $78-82^{\circ} \mathrm{C}$. ${ }^{1} \mathrm{H}-\mathrm{NMR}$ $\left(\mathrm{CDCl}_{3}, 400 \mathrm{MHz}\right): \delta=8.03(\mathrm{~d}, \mathrm{~J}=8.2 \mathrm{~Hz}, 2 \mathrm{H}, \mathrm{Ar}-\mathrm{H}), 7.94(\mathrm{~d}, \mathrm{~J}=8.5 \mathrm{~Hz}, 2 \mathrm{H}, \mathrm{Ar}-\mathrm{H}), 7.68$ (t, J = 8.2 Hz, 2H, Ar-H), $7.53(\mathrm{t}, \mathrm{J}=8.2 \mathrm{~Hz}, 2 \mathrm{H}, \mathrm{Ar}-\mathrm{H}), 7.15(\mathrm{~s}, 2 \mathrm{H}, \mathrm{Ar}-\mathrm{H}), 5.02$ (s, b, 2H, $\mathrm{OH}), 4.87$ (dd, J = 7.4, 3.9 Hz, 2H, CH), $2.66\left(\mathrm{~s}, 6 \mathrm{H}, \mathrm{CH}_{3}\right), 1.99\left(\mathrm{~m}, 2 \mathrm{H}, \mathrm{CH}_{2}\right), 1.79(\mathrm{~m}$, $\left.2 \mathrm{H}, \mathrm{CH}_{2}\right), 1.67\left(\mathrm{~m}, 2 \mathrm{H}, \mathrm{CH}_{2}\right) \cdot{ }^{13} \mathrm{C}-\mathrm{NMR}\left(\mathrm{CDCl}_{3}, 400 \mathrm{~Hz}\right): \delta=161.78,146.19,145.12$, 129.35, 129.29, 127.50, 126.06, 123.73, 118.93, 72.53, 38.10, 21.19, 18.89. ESI-MS: $\mathrm{m} / \mathrm{z} 387.5\left(\mathrm{M}+\mathrm{H}^{+}\right)$; calculated: 387.2.

(1S,5S)-1,5-bis(4-methylquinol-2-yl)pentane-1,5-diyl dimethanesulfonate (10):

To a solution of methanesulfonyl chloride $(2.66 \mathrm{~g}, 23.2 \mathrm{mmol})$ in $70 \mathrm{~mL}$ of methylene chloride was added slowly $140 \mathrm{~mL}$ of methylene chloride solution of diol 9 $(2.24 \mathrm{~g}, 5.8 \mathrm{mmol})$ and dry triethylamine $(4.72 \mathrm{~g}, 46.4 \mathrm{mmol})$ at $-35^{\circ} \mathrm{C}$. The mixture was stirred at $-35^{\circ} \mathrm{C}$ for $3 \mathrm{~h}$. After extractive isolation with methylene chloride and water, column chromatography (silica gel pretreated with triethylamine, methylene chloride / ethyl acetate $4: 1$ ) was used to purify the product. A white solid (2.74 $\mathrm{g}, 87 \%$ ) was obtained. Melting point: $123-125^{\circ} \mathrm{C} .{ }^{1} \mathrm{H}-\mathrm{NMR}\left(\mathrm{CDCl}_{3}, 400 \mathrm{MHz}\right): \delta=8.03(\mathrm{~d}, \mathrm{~J}=8.3 \mathrm{~Hz}$, 2H, Ar-H), 7.99 (d, J = 8.2 Hz, 2H, Ar-H), 7.72 (t, J = $7.4 \mathrm{~Hz}, 2 \mathrm{H}, \operatorname{Ar}-\mathrm{H}), 7.58$ (t, J = 7.4 $\mathrm{Hz}, 2 \mathrm{H}, \mathrm{Ar}-\mathrm{H}), 7.38$ (s, 2H, Ar-H), 5.71 (dd, J = 7.6, 5.7 Hz, 2H, CH), $2.88\left(\mathrm{~s}, 6 \mathrm{H}, \mathrm{CH}_{3}\right.$ ), $2.72\left(\mathrm{~s}, 6 \mathrm{H}, \mathrm{CH}_{3}\right), 2.24\left(\mathrm{~m}, 2 \mathrm{H}, \mathrm{CH}_{2}\right), 2.15\left(\mathrm{~m}, 2 \mathrm{H}, \mathrm{CH}_{2}\right), 1.67\left(\mathrm{~m}, 2 \mathrm{H}, \mathrm{CH}_{2}\right) \cdot{ }^{13} \mathrm{C}-\mathrm{NMR}$ $\left(\mathrm{CDCl}_{3}, 400 \mathrm{~Hz}\right): \delta=157.34,147.16,146.09,129.94,129.71,127.83,126.87,123.81$, 118.91, 84.57, 38.65, 35.02, 21.11, 18.98. ESI-MS: $\mathrm{m} / \mathrm{z} 543.2\left(\mathrm{M}+\mathrm{H}^{+}\right)$; calculated: 543.1. Anal. Calcd (mass\%) for $\mathrm{C}_{27} \mathrm{H}_{30} \mathrm{~N}_{2} \mathrm{O}_{6} \mathrm{~S}_{2}$ : C, 59.76; $\mathrm{H}, 5.57 ; \mathrm{N}, 5.16$. Found: $\mathrm{C}$, $59.94 ; H, 5.36 ;$ N, 5.16. 
2,2'-((2R,6R)-1-((4-Methylquinolin-2-yl)methyl)piperidine-2,6-diyl)bis(4-methylquinoline) (1):

Dimesylate $10(0.163 \mathrm{~g}, 0.3 \mathrm{mmol})$ was dissolved in amine $6(2.07 \mathrm{~g}, 12.0 \mathrm{mmol})$ at $0^{\circ} \mathrm{C}$ in a pear-shaped flask equipped with a magnetic stir bar. The mixture solidified after 30 min of stirring, and was kept at room temperature for $3 \mathrm{~h}$. A silica gel column was employed to separate the crude product from the excess amine 6 . The crude product was collected using $1 \% \mathrm{MeOH}$ in methylene chloride as eluent, and amine 6 was recovered using $7 \% \mathrm{MeOH}$ in methylene chloride (the recovery rate was $86 \%$ ). The crude product was subjected to HPLC purification (semi-prep C18 column, $\mathrm{CH}_{3} \mathrm{CN} / \mathrm{H}_{2} \mathrm{O}$ from $70: 30$ to $100: 0)$. A white solid product was collected $(0.126 \mathrm{~g})$ with a yield of $80 \%$. The de for the product was $95 \%$ as determined by ${ }^{1} \mathrm{H}-\mathrm{NMR}$ spectrum, and ee for the trans compound was $>99 \%$ as determined by HPLC using a chiral column (ChiralCel, OJ-H 0.46cm x $25 \mathrm{~cm}, 20: 80$ acetonitrile / isopropanol (with 1\% triethylamine)). Melting point: $98-100^{\circ} \mathrm{C} .{ }^{1} \mathrm{H}-\mathrm{NMR}\left(\mathrm{CD}_{3} \mathrm{CN}, 400 \mathrm{MHz}\right): \delta=8.09(\mathrm{~d}, \mathrm{~J}=8.3 \mathrm{~Hz}, 2 \mathrm{H}, \mathrm{Ar}-\mathrm{H}), 8.03(\mathrm{~d}$, $\mathrm{J}=8.3 \mathrm{~Hz}, 2 \mathrm{H}, \operatorname{Ar}-\mathrm{H}), 7.99(\mathrm{~d}, \mathrm{~J}=8.3 \mathrm{~Hz}, 1 \mathrm{H}, \operatorname{Ar}-\mathrm{H}), 7.75(\mathrm{~m}, 2 \mathrm{H}, \operatorname{Ar}-\mathrm{H}), 7.68(\mathrm{~d}, \mathrm{~J}=8.4$ $\mathrm{Hz}, 1 \mathrm{H}, \mathrm{Ar}-\mathrm{H}), 7.62-7.49(\mathrm{~m}, 7 \mathrm{H}, \mathrm{Ar}-\mathrm{H}), 4.85(\mathrm{dd}, \mathrm{J}=6.6,4.8 \mathrm{~Hz}, 2 \mathrm{H}, \mathrm{CH}), 3.89(\mathrm{~d}, \mathrm{~J}=$ $\left.14.8 \mathrm{~Hz}, 1 \mathrm{H}, \mathrm{CH}_{2}\right), 3.85\left(\mathrm{~d}, \mathrm{~J}=14.8 \mathrm{~Hz}, 1 \mathrm{H}, \mathrm{CH}_{2}\right), 2.64\left(\mathrm{~s}, 9 \mathrm{H}, \mathrm{CH}_{3}\right), 2.24-2.06(\mathrm{~m}, 4 \mathrm{H}$, $\left.\mathrm{CH}_{2}\right), 1.90-1.82\left(\mathrm{~m}, 2 \mathrm{H}, \mathrm{CH}_{2}\right) .{ }^{13} \mathrm{C}-\mathrm{NMR}\left(\mathrm{CD}_{3} \mathrm{CN}, 400 \mathrm{~Hz}\right): \delta=164.16,160.58,146.83$, $143.88,129.17,128.69,128.57,128.35,126.72,125.43,125.24,123.53,123.41$, 121.33, 62.42, 57.18, 29.40, 18.59, 17.49. ESI-MS: $\mathrm{m} / \mathrm{z} 523.2\left(\mathrm{M}+\mathrm{H}^{+}\right), 262.2$ $\left(\left(\mathrm{M}+2 \mathrm{H}^{+}\right) / 2\right)$; calculated: 523.3 , 262.2. Anal. Calcd (mass\%) for $\mathrm{C}_{36} \mathrm{H}_{34} \mathrm{~N}_{4}$ : C, 82.72; $\mathrm{H}$, 6.56, N, 10.72. Found: C, 82.33; H, 6.36; N, 10.62 . 
$\mathrm{Cu}^{\prime}(\mathbf{1}) \mathrm{ClO}_{4}:$

Compound $1(3.1 \mathrm{mg}, 0.006 \mathrm{mmol})$ and $\mathrm{Cu}^{\prime}\left(\mathrm{CH}_{3} \mathrm{CN}\right){ }_{4} \mathrm{ClO}_{4}(5.0 \mathrm{mg}, 0.013 \mathrm{mmol})$ were mixed in $0.7 \mathrm{~mL} \mathrm{CD}_{3} \mathrm{CN}$ inside a glove box and the solution was allowed to stand for 30 min. The filtrate was used for NMR spectra. Solutions for CD and UV spectroscopy were prepared in similar fashion in degassed dry $\mathrm{CH}_{3} \mathrm{CN} .{ }^{1} \mathrm{H}-\mathrm{NMR}$ $\left(\mathrm{CD}_{3} \mathrm{CN}, 400 \mathrm{MHz}\right): \delta=8.76(\mathrm{~d}, \mathrm{~J}=8.5 \mathrm{~Hz}, 1 \mathrm{H}, \operatorname{Ar}-\mathrm{H}), 8.49(\mathrm{~d}, \mathrm{~J}=8.4 \mathrm{~Hz}, 1 \mathrm{H}, \operatorname{Ar}-\mathrm{H})$, $8.30(\mathrm{~d}, \mathrm{~J}=8.3 \mathrm{~Hz}, 1 \mathrm{H}, \mathrm{Ar}-\mathrm{H}), 8.17-8.11(\mathrm{~m}, 3 \mathrm{H}, \mathrm{Ar}-\mathrm{H}), 8.03(\mathrm{t}, \mathrm{J}=7.4 \mathrm{~Hz}, 1 \mathrm{H}, \mathrm{Ar}-\mathrm{H})$, $7.87(\mathrm{t}, \mathrm{J}=7.3 \mathrm{~Hz}, 1 \mathrm{H}, \mathrm{Ar}-\mathrm{H}), 7.80(\mathrm{t}, \mathrm{J}=7.4 \mathrm{~Hz}, 1 \mathrm{H}, \mathrm{Ar}-\mathrm{H}), 7.74$ (t, J = $7.5 \mathrm{~Hz}, 1 \mathrm{H}, \mathrm{Ar}-$ H), 7.67 (t, 7.2 Hz, 1H, Ar-H), 7.64 (t, J = 7.3 Hz, 1H Ar-H), 7.63 (s, 1H, Ar-H), 7.15 (s, 1H, Ar-H), $6.88(\mathrm{~s}, 1 \mathrm{H}, \mathrm{Ar}-\mathrm{H}), 6.03(\mathrm{~m}, 1 \mathrm{H}, \mathrm{CH}), 4.58(\mathrm{~m}, 1 \mathrm{H}, \mathrm{CH}), 4.13(\mathrm{~d}, \mathrm{~J}=16.6 \mathrm{~Hz}$, $1 \mathrm{H}, \mathrm{CH}_{2}$ ), $3.77\left(\mathrm{~d}, \mathrm{~J}=16.5 \mathrm{~Hz}, 1 \mathrm{H}, \mathrm{CH}_{2}\right), 2.79\left(\mathrm{~s}, 3 \mathrm{H}, \mathrm{CH}_{3}\right), 2.70\left(\mathrm{~s}, 3 \mathrm{H}, \mathrm{CH}_{3}\right), 2.57(\mathrm{~s}$, $\left.3 \mathrm{H}, \mathrm{CH}_{3}\right), 2.37-1.67\left(\mathrm{~m}, 6 \mathrm{H}, \mathrm{CH}_{2}\right) .{ }^{13} \mathrm{C}-\mathrm{NMR}\left(\mathrm{CD}_{3} \mathrm{CN}, 400 \mathrm{~Hz}\right): \delta=163.20,161.92$, $158.90,147.74,147.36,146.52,146.46,145.21,130.62,130.17,129.50,128.46$, $128.24,127.95,127.80,126.69,126.55,126.03,124.27,123.80,121.99,121.90$, 121.35, 63.36, 61.23, 57.40, 33.59, 30.09, 17.84, 17.60, 17.52, 17.15. MALDI-MS: m/z $585.3\left(\mathrm{M}+\mathrm{H}^{+}\right)$; calculated: 585.2.

$\mathrm{Cu}^{\prime \prime}(\mathbf{1})\left(\mathrm{ClO}_{4}\right)_{2}$ :

The samples for CD and UV spectroscopy were prepared in situ by mixing 1:1 ligand and $\mathrm{Cu}^{\prime \prime}\left(\mathrm{ClO}_{4}\right)_{2}$ stock solutions. MALDI-MS: m/z 585.4 (M+H'); calculated: 585.2.

$\mathrm{Zn}^{\prime \prime}(1)\left(\mathrm{ClO}_{4}\right)_{2}$ :

Compound $1(3.5 \mathrm{mg}, 0.007 \mathrm{mmol})$ and $\mathrm{Zn}^{\prime \prime}\left(\mathrm{ClO}_{4}\right)_{2}(14 \mu \mathrm{L}$ of $0.5 \mathrm{M}, 0.007 \mathrm{mmol})$ were mixed in $2 \mathrm{~mL}$ of $\mathrm{CH}_{3} \mathrm{CN}$. The solvent was removed and the residue was coevaporated with dry $\mathrm{CH}_{3} \mathrm{CN}$. The residue was dissolved in $0.7 \mathrm{~mL} \mathrm{CD}{ }_{3} \mathrm{CN}$ for NMR 
analysis. The samples for CD and UV spectroscopy were made in situ by mixing 1:1 ligand and $\mathrm{Zn}^{\prime \prime}\left(\mathrm{ClO}_{4}\right)_{2}$ stock solutions. ${ }^{1} \mathrm{H}-\mathrm{NMR}\left(\mathrm{CD}_{3} \mathrm{CN}, 400 \mathrm{MHz}\right): \delta=8.53(\mathrm{~d}, \mathrm{~J}=8.5$ $\mathrm{Hz}, 1 \mathrm{H}, \operatorname{Ar}-\mathrm{H}), 8.41(\mathrm{~d}, \mathrm{~J}=8.4 \mathrm{~Hz}, 1 \mathrm{H}, \operatorname{Ar}-\mathrm{H}), 8.20(\mathrm{~d}, \mathrm{~J}=8.1 \mathrm{~Hz}, 1 \mathrm{H}, \operatorname{Ar}-\mathrm{H}), 8.14-8.11$ (m, 3H, Ar-H), $7.98(\mathrm{t}, \mathrm{J}=7.4 \mathrm{~Hz}, 1 \mathrm{H}, \mathrm{Ar}-\mathrm{H}), 7.92(\mathrm{t}, \mathrm{J}=7.5 \mathrm{~Hz}, 1 \mathrm{H}, \operatorname{Ar}-\mathrm{H}), 7.80-7.68(\mathrm{~m}$, 4H, Ar-H), 7.62 (t, J = 7.5 Hz, 1H, Ar-H), 7.54 (s, 1H, Ar-H), 7.30 (s, 1H, Ar-H), 4.71 (d, J $=10.4 \mathrm{~Hz}, 1 \mathrm{H}, \mathrm{CH}), 4.64\left(\mathrm{~d}, \mathrm{~J}=18.0 \mathrm{~Hz}, 1 \mathrm{H}, \mathrm{CH}_{2}\right), 4.54(\mathrm{~s}, 1 \mathrm{H}, \mathrm{CH}), 4.07(\mathrm{~d}, \mathrm{~J}=18.0$ $\left.\mathrm{Hz}, 1 \mathrm{H}, \mathrm{CH}_{2}\right), 2.80\left(\mathrm{~s}, 3 \mathrm{H}, \mathrm{CH}_{3}\right), 2.75\left(\mathrm{~s}, 3 \mathrm{H}, \mathrm{CH}_{3}\right), 2.67\left(\mathrm{~s}, 3 \mathrm{H}, \mathrm{CH}_{3}\right), 2.66-1.62(\mathrm{~m}, 6 \mathrm{H}$, $\left.\mathrm{CH}_{2}\right) \cdot{ }^{13} \mathrm{C}-\mathrm{NMR}\left(\mathrm{CD}_{3} \mathrm{CN}, 400 \mathrm{~Hz}\right): \delta=159.50,158.64,157.64,152.37,152.15,151.37$, $144.72,144.24,143.82,131.92,131.80,131.07,128.22,128.09,127.64,127.57$ $127.47,127.14,125.96,125.09,124.98,124.86,121.74,120.13,119.68,61.05,59.91$, $52.11,21.02,20.37,19.67,18.24,18.04$

\section{References:}

a. Dai, Z.; Proni, G.; Mancheno, D.; Karimi, S.; Berova, N.; Canary, J. W. J. Am. Chem. Soc. 2004, 126, 11760-11761.

Table S1. Optical properties of $\mathbf{1}, \mathrm{Cu}^{\mathrm{I}}(\mathbf{1}) \mathrm{ClO}_{4}$ and $\mathrm{Cu}^{\mathrm{II}}(\mathbf{1})\left(\mathrm{ClO}_{4}\right)_{2}$.

\begin{tabular}{|c|c|c|c|c|c|c|}
\hline & \multicolumn{2}{|r|}{ UV } & \multicolumn{4}{|c|}{$\mathrm{CD}$} \\
\hline & $\begin{array}{l}\lambda_{\max } \\
(\mathrm{nm})\end{array}$ & $\begin{array}{l}\Delta \varepsilon \\
\left(\mathrm{L} \cdot \mathrm{cm}^{-1} \cdot \mathrm{mol}^{-1}\right)\end{array}$ & $\begin{array}{l}\lambda_{\text {CE1 }} \\
(\mathrm{nm})\end{array}$ & $\begin{array}{l}\Delta \varepsilon_{\mathrm{CE} 1} \\
\left(\mathrm{~L} \cdot \mathrm{cm}^{-1} \cdot \mathrm{mol}^{-1}\right)\end{array}$ & $\begin{array}{l}\lambda_{\mathrm{CE} 2} \\
(\mathrm{~nm})\end{array}$ & $\begin{array}{l}\Delta \varepsilon_{\mathrm{CE} 2} \\
\left(\mathrm{~L} \cdot \mathrm{cm}^{-1} \cdot \mathrm{mol}^{-1}\right)\end{array}$ \\
\hline 1 & 228 & $1.34 \times 10^{5}$ & & & 231 & +199 \\
\hline $\mathrm{Cu}^{\mathrm{I}}(\mathbf{1}) \mathrm{ClO}_{4}$ & 229 & $1.45 \times 10^{5}$ & 239 & -63 & 230 & +204 \\
\hline $\mathrm{Cu}^{\mathrm{II}}(\mathbf{1})\left(\mathrm{ClO}_{4}\right)_{2}$ & $\begin{array}{l}238 / \\
227\end{array}$ & $\begin{array}{l}8.3 \times 10^{4} / \\
1.23 \times 10^{5}\end{array}$ & 239 & +687 & 227 & -407 \\
\hline
\end{tabular}




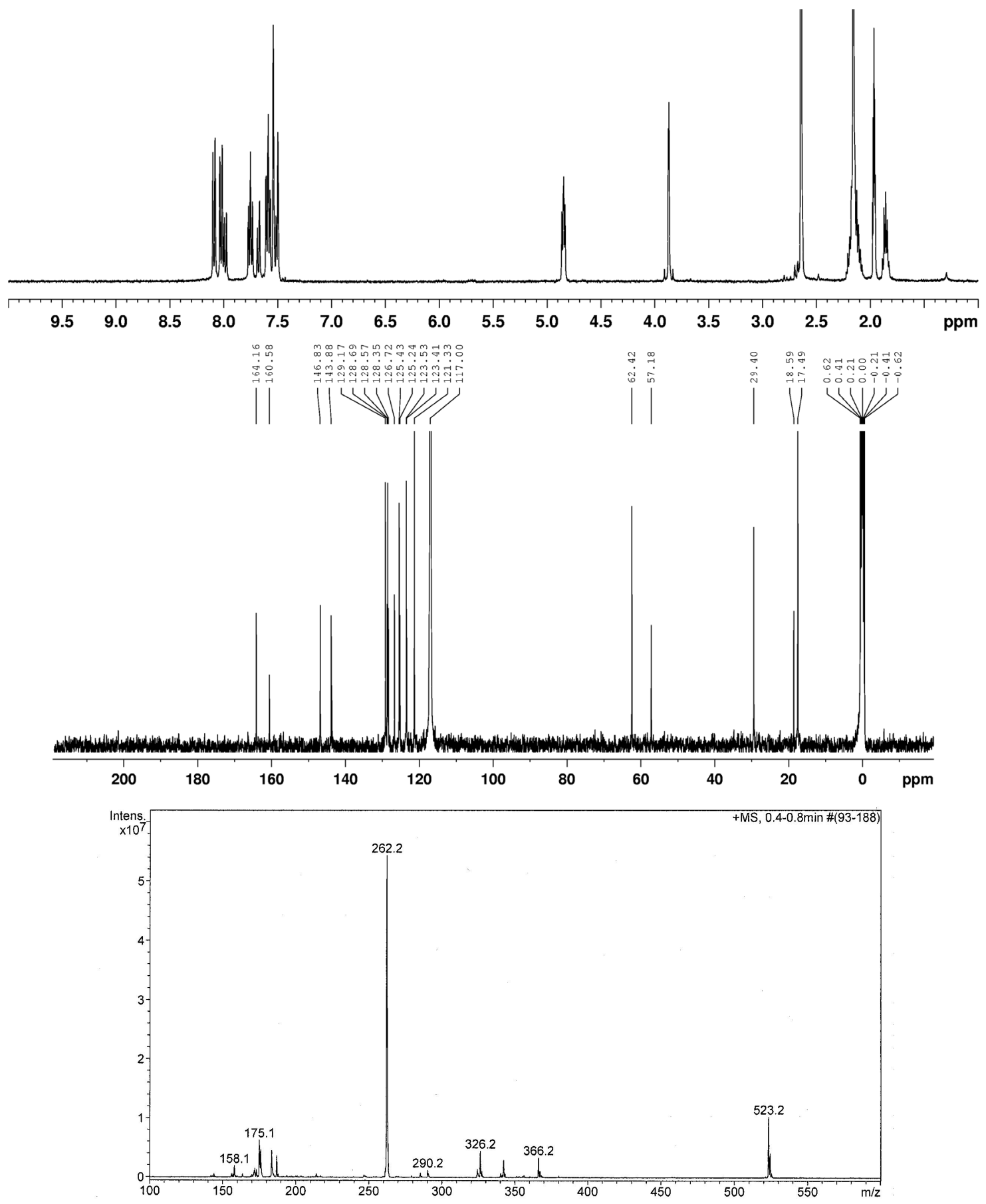

Figure S1. ${ }^{1} \mathrm{H}-\mathrm{NMR}$ (in $\mathrm{CD}_{3} \mathrm{CN}$ ), ${ }^{13} \mathrm{C}-\mathrm{NMR}$ (in $\mathrm{CD}_{3} \mathrm{CN}$ ) and ESI-MS spectra of compound 1. 


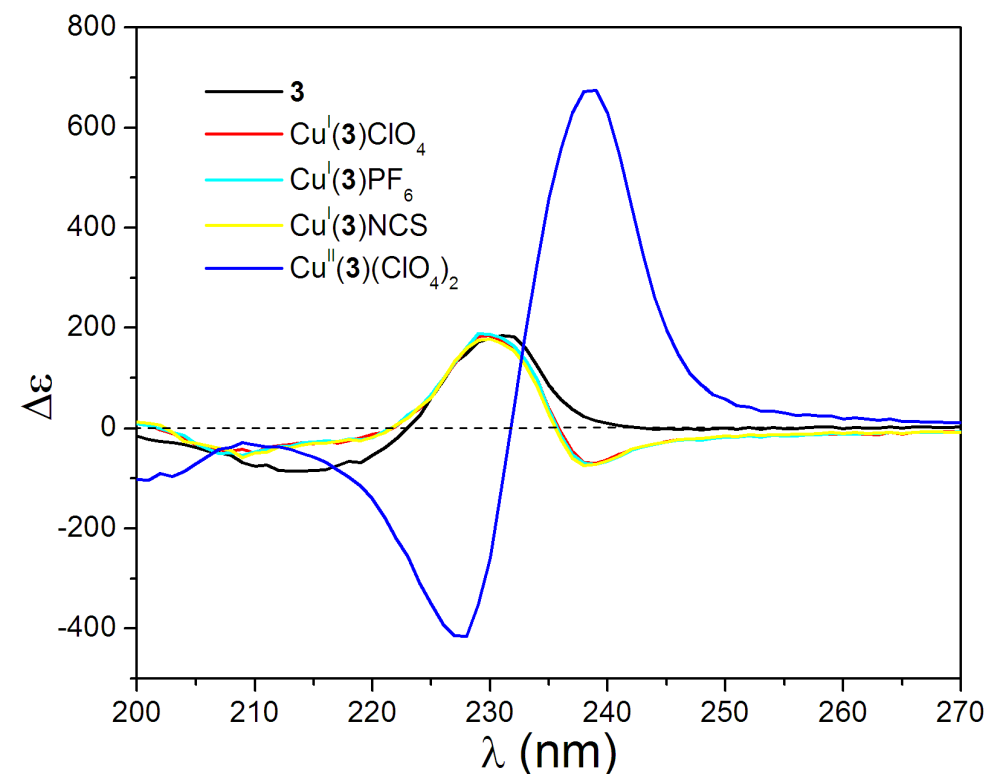

Figure S2. CD spectra of $\mathrm{Cu}^{\prime \prime}$ and $\mathrm{Cu}^{\prime}$ complexes with various counter ions in acetonitrile. The concentrations are $50 \mu \mathrm{M}$, and the units of $\Delta \varepsilon$ are $\mathrm{L} \cdot \mathrm{cm}^{-1} \cdot \mathrm{mol}^{-1}$.

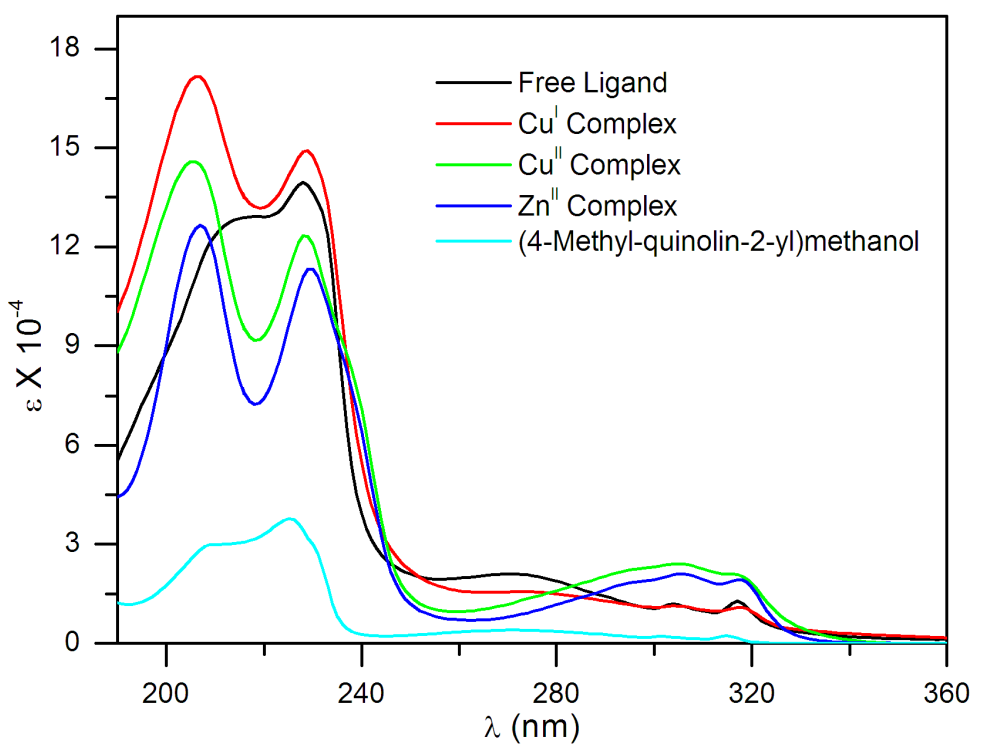

Figure S3. UV spectra of $\mathbf{1}$ (black), $\mathrm{Cu}^{\prime}(\mathbf{1}) \mathrm{ClO}_{4}$ (red), $\mathrm{Cu}^{\prime \prime}(\mathbf{1})\left(\mathrm{ClO}_{4}\right)_{2}$ (blue) (green), $\mathrm{Zn}^{\prime \prime}(\mathbf{1})\left(\mathrm{ClO}_{4}\right)_{2}$ (blue), and (4-methyl-quinolin-2-yl)methanol representing one arm (cyan). The unit of $\varepsilon$ is $\mathrm{L} \cdot \mathrm{cm}^{-}$ ${ }^{1} \cdot \mathrm{mol}^{-1}$. 


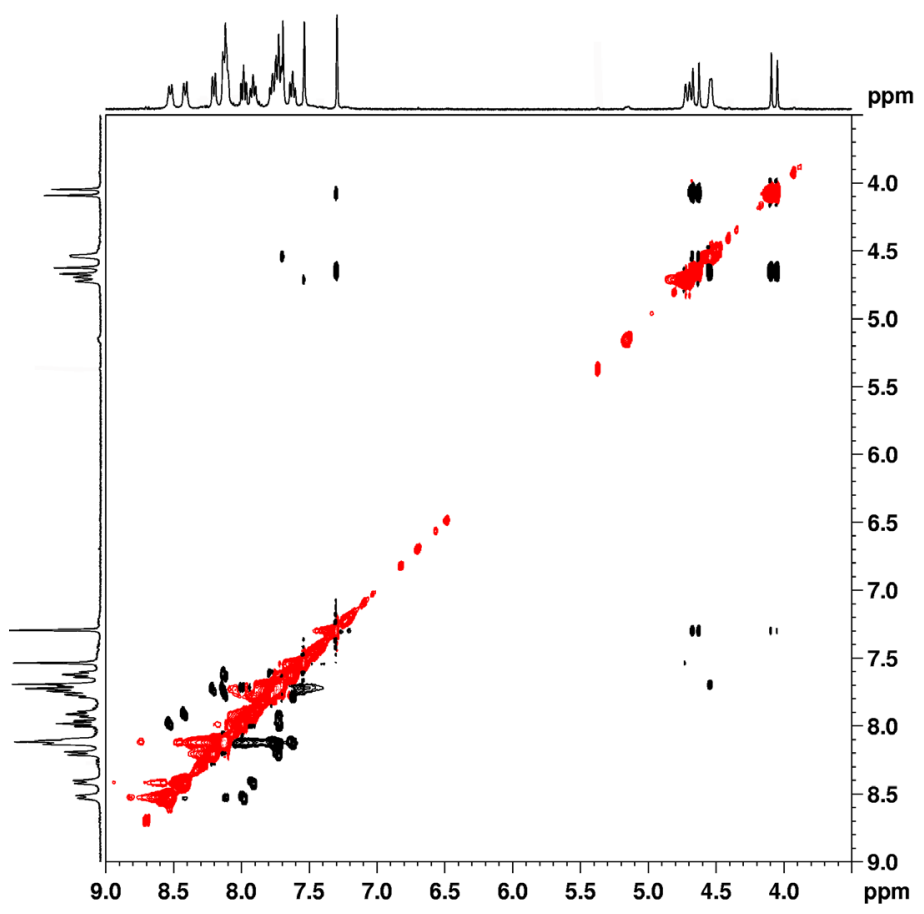

Figure S4. NOESY spectrum of $\mathrm{Zn}^{\prime \prime}(\mathbf{1})\left(\mathrm{ClO}_{4}\right)_{2}$ in $\mathrm{CD}_{3} \mathrm{CN}$.

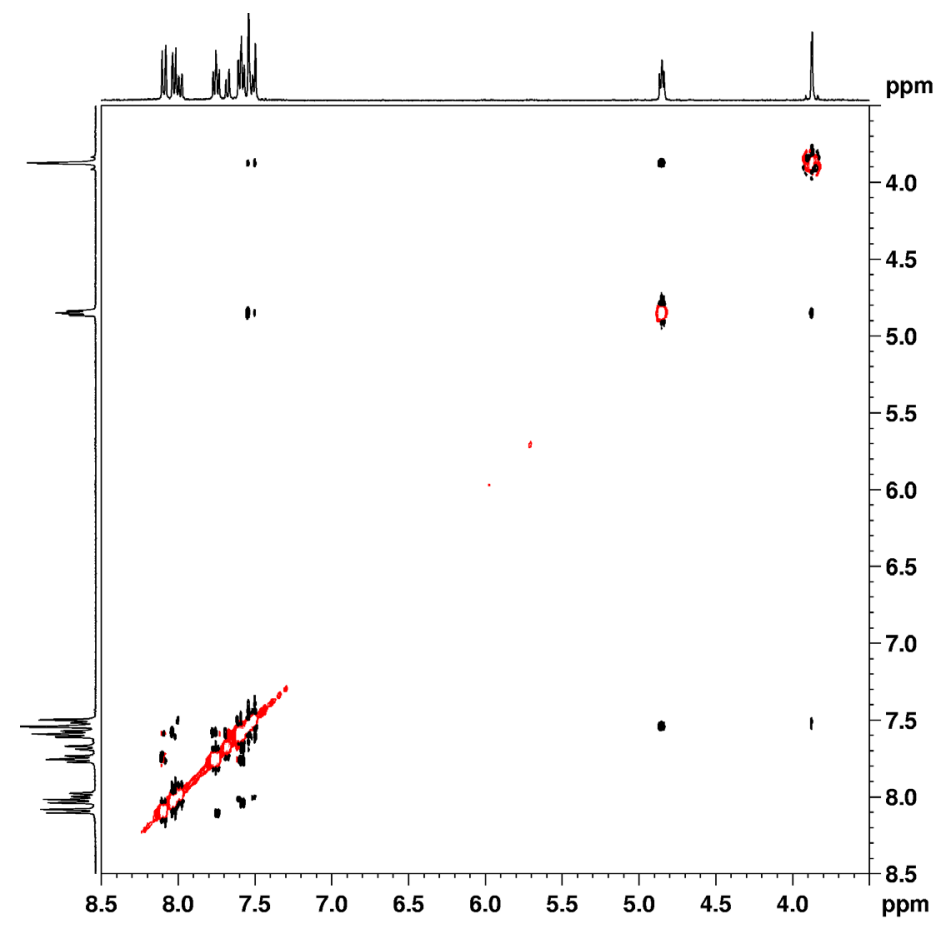

Figure S5. NOESY spectrum of 1 in $\mathrm{CD}_{3} \mathrm{CN}$. 


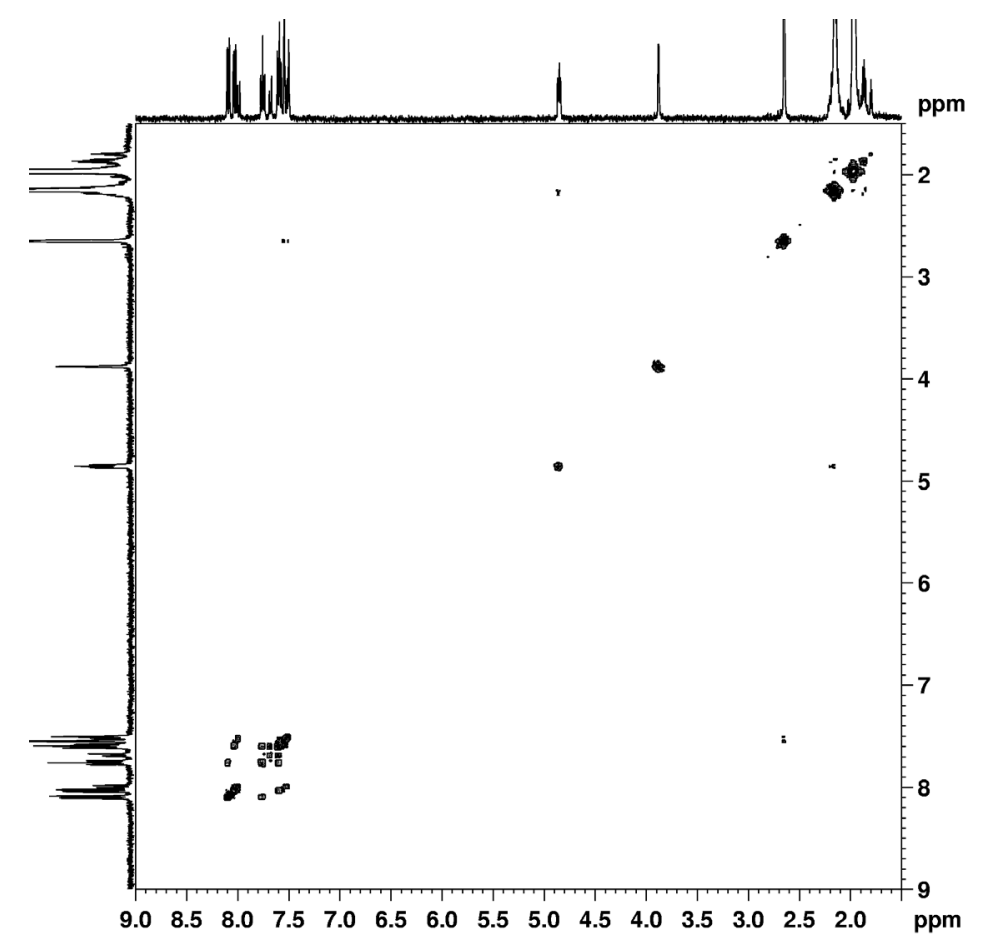

Figure S6. COSY spectrum of 1 in $\mathrm{CD}_{3} \mathrm{CN}$.

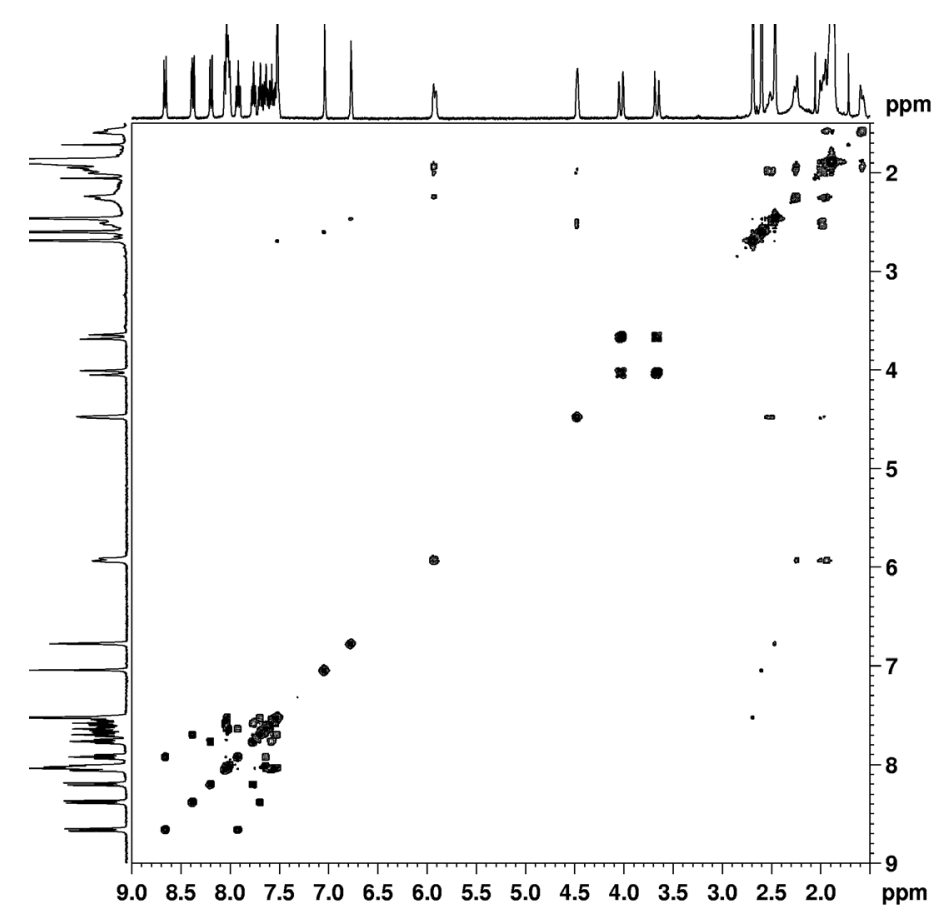

Figure S7. COSY spectrum of $\mathrm{Cu}^{\prime}(\mathbf{1}) \mathrm{ClO}_{4}$ in $\mathrm{CD}_{3} \mathrm{CN}$. 


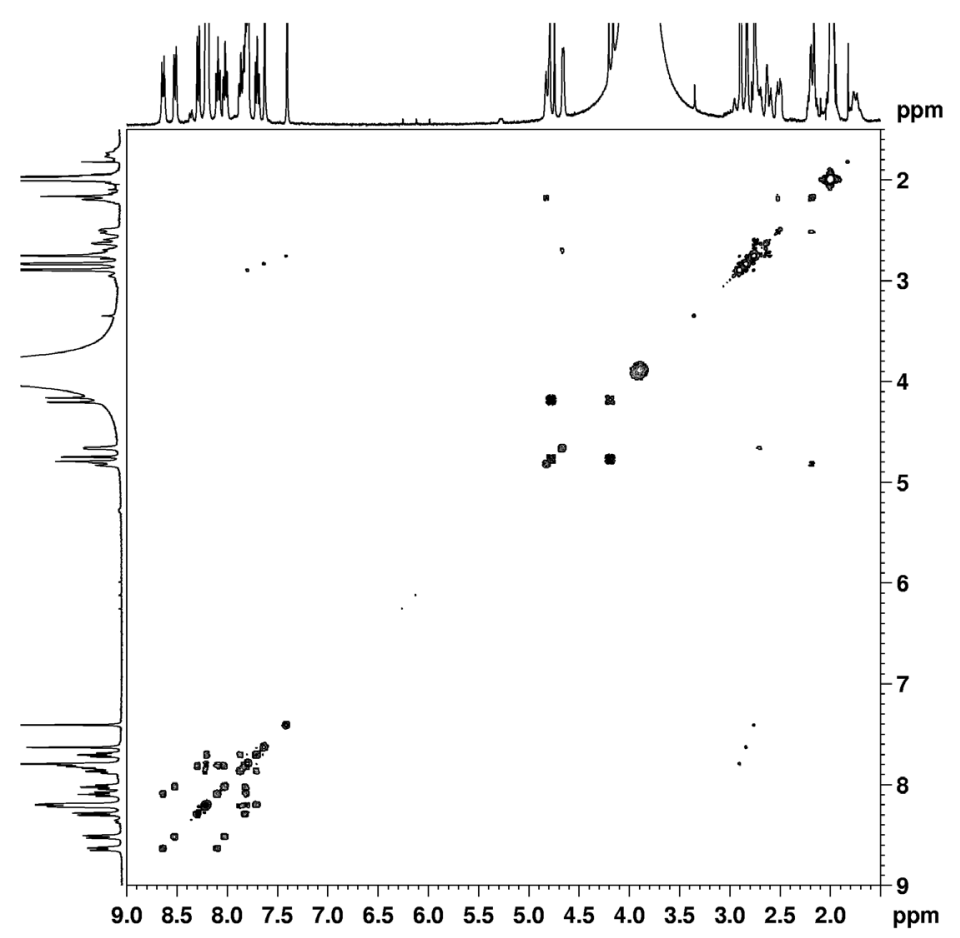

Figure S8. COSY spectrum of $\mathrm{Zn}^{\prime \prime}(\mathbf{1})\left(\mathrm{ClO}_{4}\right)_{2}$ in $\mathrm{CD}_{3} \mathrm{CN}$.

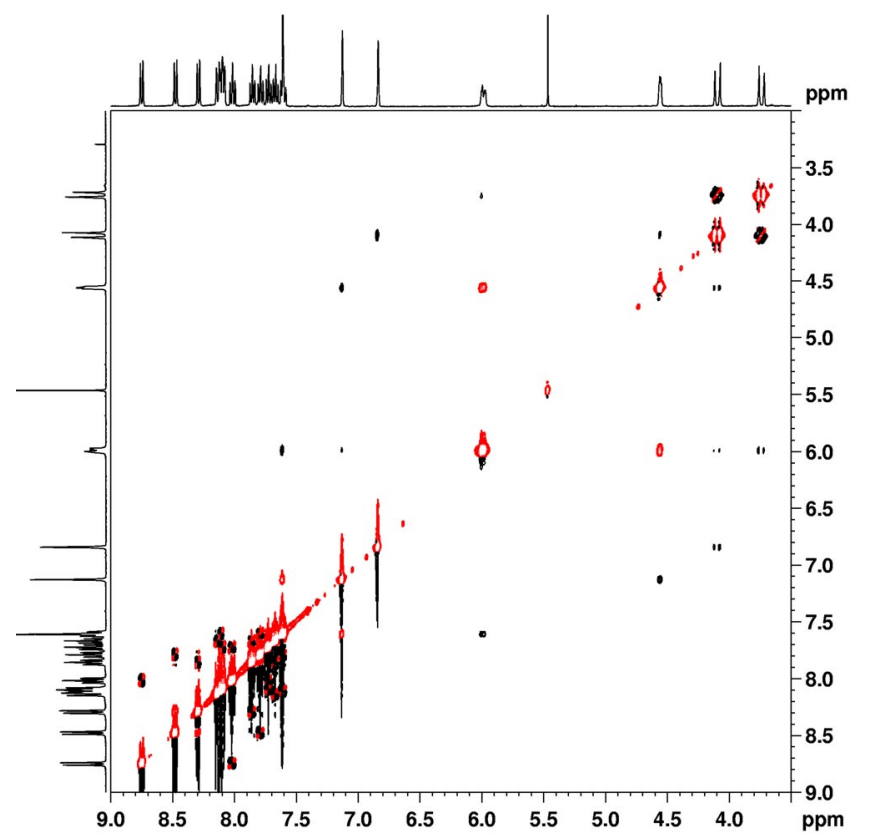

Figure S10. ROESY spectrum of $\mathrm{Cu}^{\prime}(\mathbf{1}) \mathrm{ClO}_{4}$ in $\mathrm{CD}_{3} \mathrm{CN}$. 


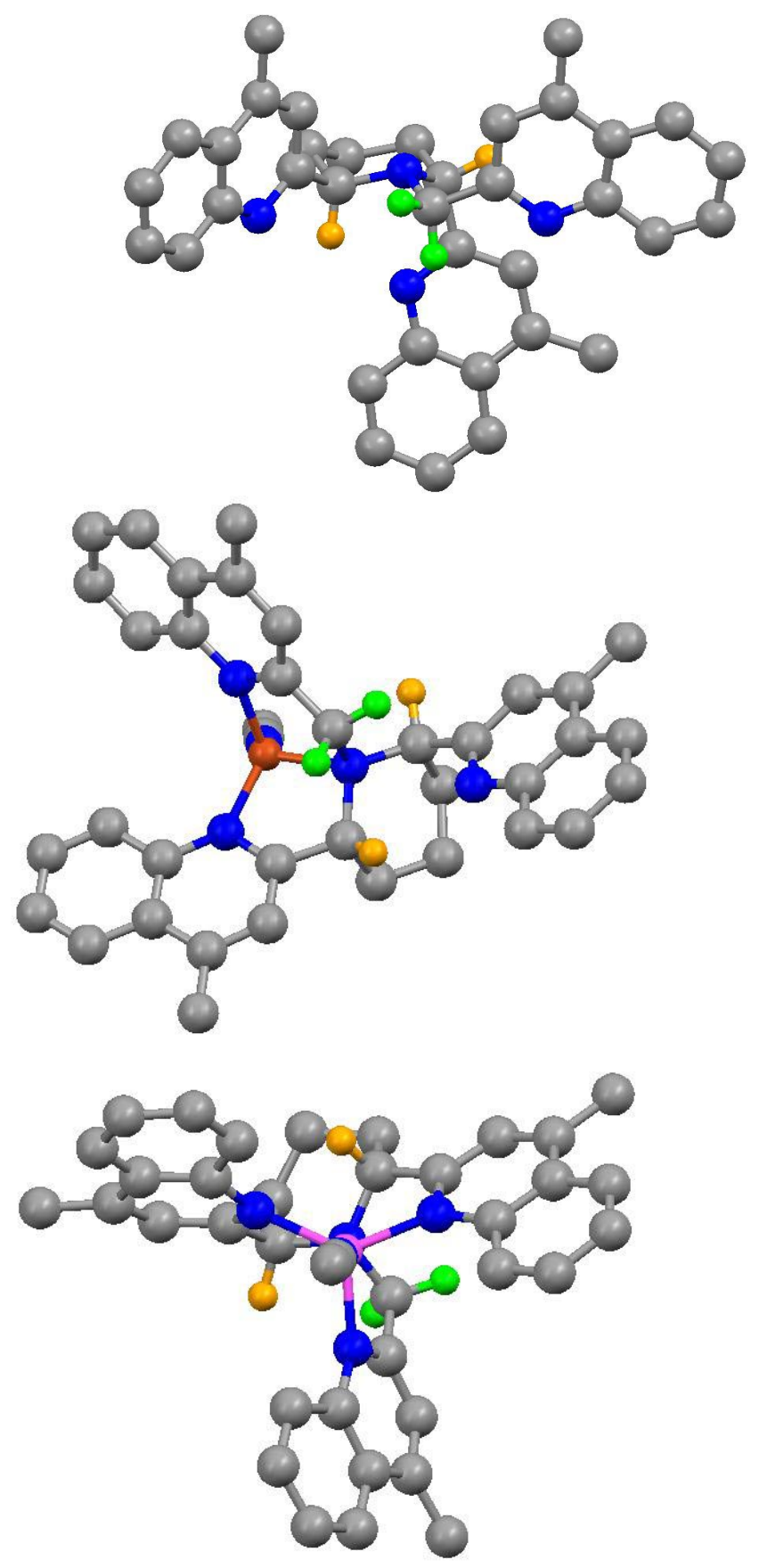

Figure S11. Calculated structures for $\mathbf{1}$ (top), $\mathrm{Cu}^{\prime}(\mathbf{1})\left(\mathrm{CH}_{3} \mathrm{CN}\right.$ ) (middle) and $\mathrm{Cu}^{\prime \prime}(\mathbf{1})\left(\mathrm{CH}_{3} \mathrm{CN}\right.$ ) (bottom). These lowest energy structures were calculated by conformer searches. The method used for the free ligand was molecular mechanics (MMFF94), and for the two copper complexes was semi-empirical (PM3(tm)). Color shown: grey, carbon; blue, nitrogen; red, copper (I); pink, copper (II); green/brown/purple, hydrogen; most protons are omitted for clarity. 


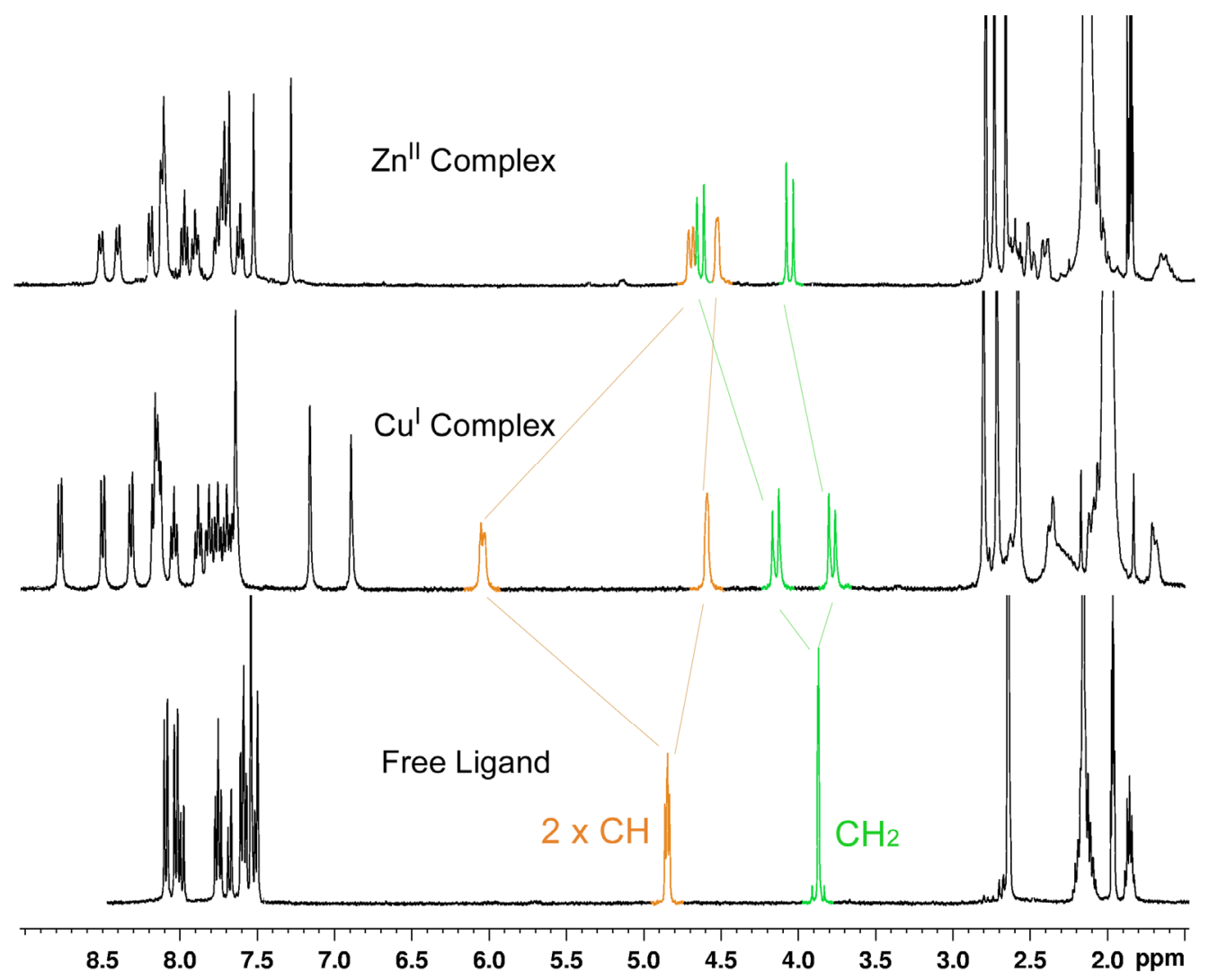

Figure S12. ${ }^{1} \mathrm{H}-\mathrm{NMR}$ spectra of 1 (bottom), $\mathrm{Cu}^{\prime}(\mathbf{1}) \mathrm{ClO}_{4}$ (middle) and $\mathrm{Zn}(\mathbf{1})\left(\mathrm{ClO}_{4}\right)_{2}$ (top) in $\mathrm{CD}_{3} \mathrm{CN}$. 